\title{
Molecular crystals on two-dimensional van der Waals substrates
}

\author{
Xiao Huang ${ }^{1 *}$ and Hua Zhang ${ }^{2 *}$
}

Two-dimensional (2D) materials, such as graphene and transition metal dichalcogenide nanosheets, have aroused enormous research interest over the past decade, because of their unique physical and chemical properties that are correlated to their atomic-scale thickness and 2D dimension [1-10]. The practical potential of 2D materials has been exploited and successfully demonstrated in various applications, such as electronics, optoelectronics, catalysis, energy storage devices and so on [1-4,11-18].

The combination of $2 \mathrm{D}$ materials and other functional structures to form hybrid architectures is promising in achieving enhanced properties and creation of new functions $[19,20]$. To date, hybrid materials that integrate $2 \mathrm{D}$ materials and inorganic crystals with desirable properties have been explored extensively, where different nanostructures of metals, metal oxides, or metal chalcogenides have been in-situ deposited on the surface of 2D materials. Especially, the orientation and surface alignment of deposited structures can be strongly affected by the crystalline lattice of 2D substrate via epitaxial growth. For example, noble metals like Pt, Pd and Ag nanostructures have been successfully deposited on $\mathrm{MoS}_{2}$ nanosheets via solution-phase epitaxial growth [21]. Additionally, semiconductor crystals have also been epitaxially deposited on $2 \mathrm{D}$ substrates via solid [22,23] or liquid-phase [24] preparation routes to yield different heterostructures. For example, GaAs thin film was deposited on pristine graphene via metal-organic chemical vapor deposition (MOCVD) [23]. The graphene substrate also acted as electrode in the obtained GaAsbased light emitting diode (LED), which can be readily transferred to any foreign substrate, such as glass, plastic and metal.

Compared with inorganic nanostructures, controlled growth of organic molecular crystals on 2D substrates has been less explored. In fact, driven by the rapid development of organic optoelectronics, great effort has been devoted to the vapor-phase deposition of organic thin films with high chemical purity, structural precision, and controllable thickness down to single-molecular level [25]. Such depo- sition requires highly crystalline substrates with clean surface, such as graphite, mica, $\mathrm{Si}(111)$ and $\mathrm{NaCl}(001)$. Similar to these bulk crystalline substrates, $2 \mathrm{D}$ crystals of layered materials can also guide organic molecules to assemble into well-ordered structures via van der Waals interactions. In the past few years, mechanically exfoliated graphene or chemical vapor deposited (CVD) graphene have been used to realize the surface assembly of organic semiconducting molecules [26,27] and oriented growth of covalent organic framework (COF) thin films [28]. Importantly, the van der Waals $2 \mathrm{D}$ substrate can enable the formation of atomically well-defined interface with organic materials for further applications [29]. This is critically essential for the development of organic electronic and optoelectronic devices.

In a recent demonstration by $\mathrm{He}$ et al. [30], dioctylbenzothienobenzothiophene $\left(\mathrm{C}_{8}\right.$-BTBT) crystals were epitaxially grown on graphene with precisely controlled thickness down to monolayer to prepare vertical organic field-effect transistors (OFETs) (Fig. 1). As suggested by discrete Fourier transform (DFT) calculations, the formation of large-area continuous single-layer $\mathrm{C}_{8}$-BTBT on graphene is energetically favored. Importantly, the charge transport in graphene is not significantly affected by the deposited $\mathrm{C}_{8}$-BTBT layer, suggesting that the organic semiconducting crystal can be regarded as quasi-freestanding with minimal disturbance from the substrate. In the same study, monolayer $\mathrm{C}_{8}$-BTBT molecular crystal was also deposited epitaxially on hexagonal boron nitride $(h-\mathrm{BN})$ to prepare a planar OFET, which showed record-high carrier mobility up to $10 \mathrm{~cm}^{2} \mathrm{~V}^{-1} \mathrm{~s}^{-1}$, due to the atomically smooth interface and weak van der Waals interaction between $h$-BN and $\mathrm{C}_{8}$-BTBT [30]. In another similar report, Lee et al. [31] demonstrated the epitaxial growth of crystalline film of rubrene on $h$-BN. The formation of atomically sharp interface with low charge trap density between rubrene and $h$-BN, combined with the use of pristine graphene as the van der Waals electrical contacts with low contact resistance led to high carrier mobilities up to $11.5 \mathrm{~cm}^{2} \mathrm{~V}^{-1} \mathrm{~s}^{-1}$ (Fig. 2), which is comparable to that of freestanding single-

Key Laboratory of Flexible Electronics \& Institute of Advanced Materials, Jiangsu National Synergistic Innovation Center for Advanced Materials, Nanjing Tech University, Nanjing 211816, China

${ }^{2}$ School of Materials Science and Engineering, Nanyang Technological University, 50 Nanyang Avenue, 639798, Singapore

*Corresponding authors (emails: hzhang@ntu.edu.sg (Zhang H); iamxhuang@njtech.edu.cn (Huang X)) 

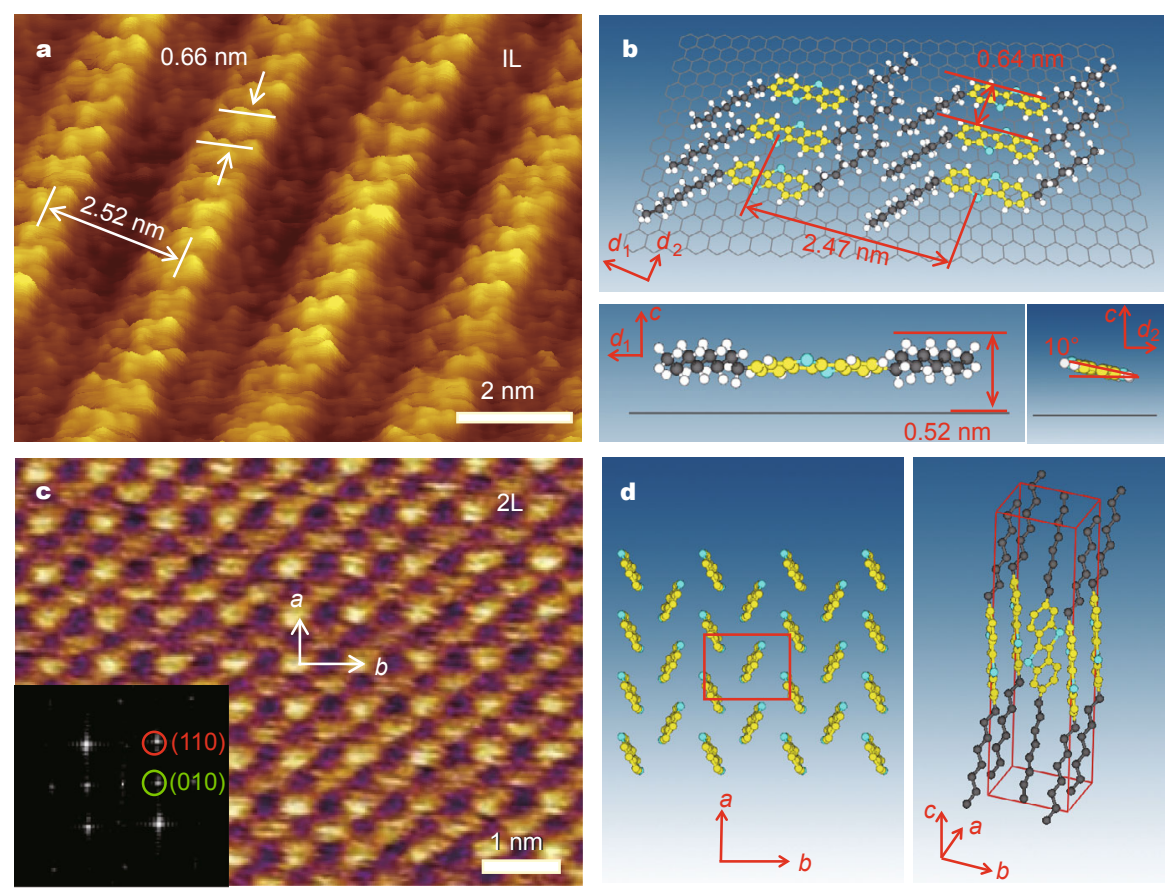

Figure 1 (a) A constant-current STM image of the initial layer of C8-BTBT on CVD-grown graphene $\left(V_{\text {sample }}=0.91 \mathrm{~V}\right.$ and $I=12.9 \mathrm{pA}$ ). (b) Top view (top panel) and side view (bottom panels) of the most stable initial layer structure obtained by DFT calculations. The lattice constants of 2.47 $\mathrm{nm}$ and $0.64 \mathrm{~nm}$ are in good agreement with experiments. (c) High-resolution AFM image of the second layer on graphene. The unit cell is marked. Inset is the Fast Fourier Transform of the AFM image with lattice indices. (d) Top view (left panel) and side view (right panel) of the $2 \mathrm{~L}$ structure according to the AFM image in (c). Reproduced from Ref. [31]. Copyright 2014, Nature Publishing Group. a
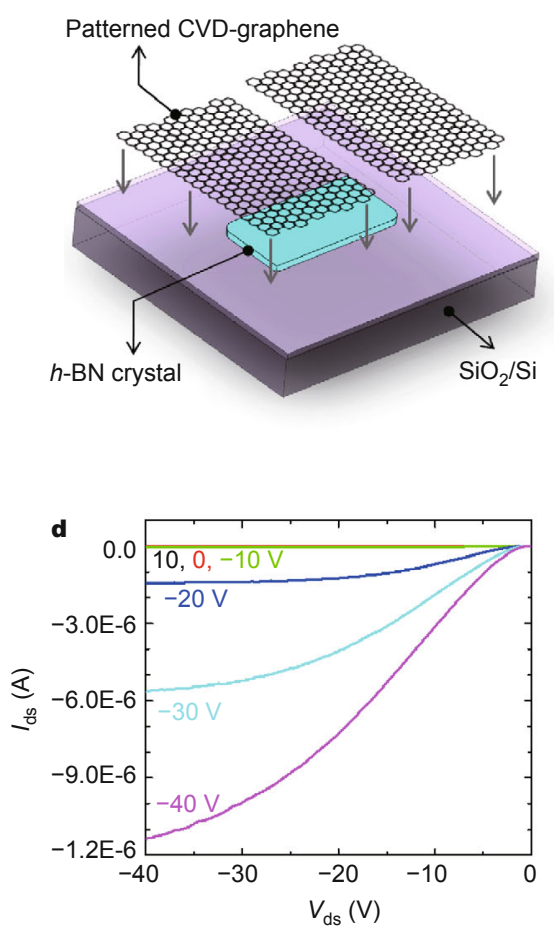

b

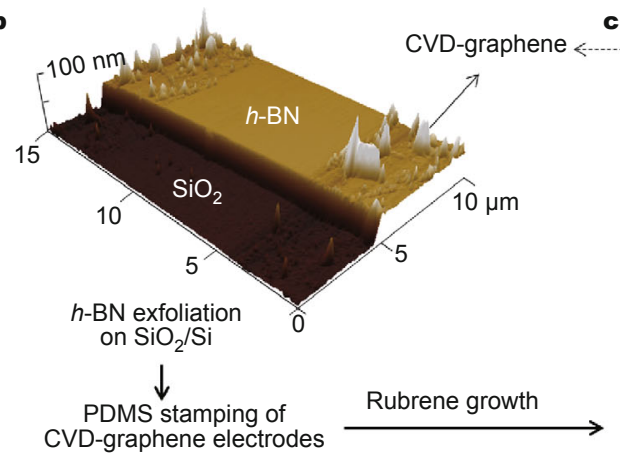

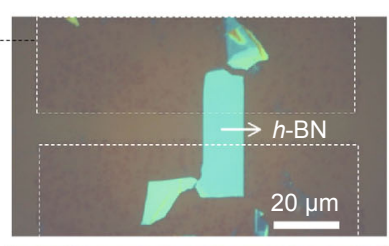
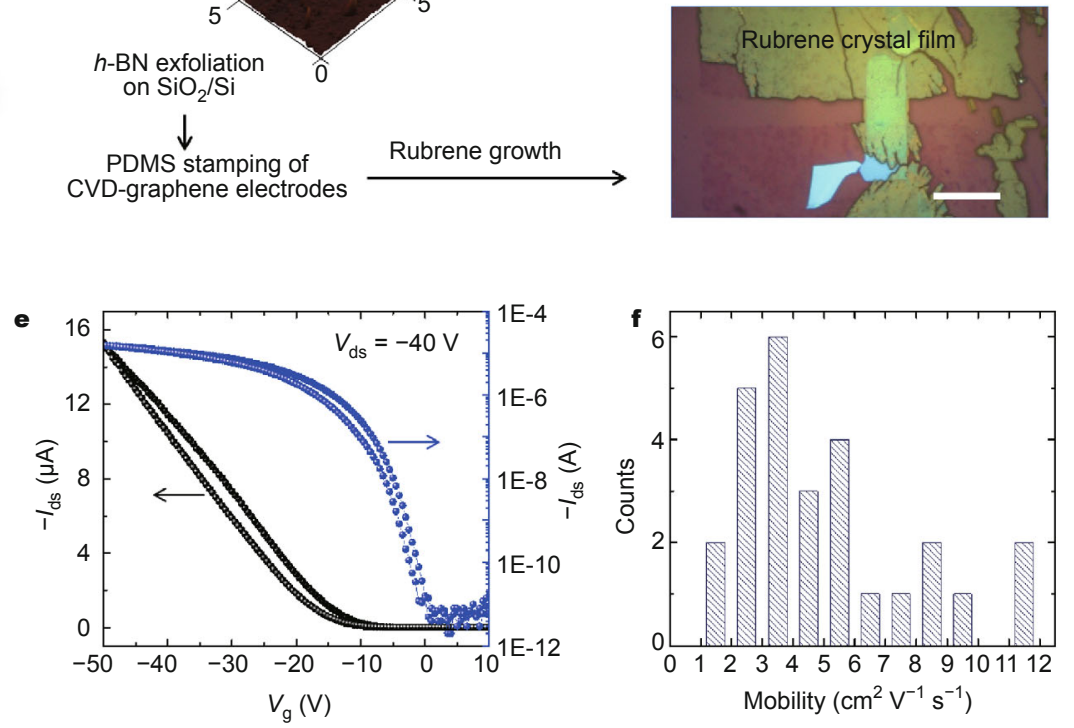

Figure 2 (a) Schematic illustration for fabrication of graphene electrodes on $h$-BN. (b) AFM image of the patterned CVD-graphene electrodes on the $h$ - $\mathrm{BN} / \mathrm{SiO}_{2}$ substrate. (c) Optical images of the device before (top) and after (bottom) rubrene crystal growth on the stacked graphene/h-BN structure. Representative output (d) and transfer (e) characteristic curves of FETs with graphene electrodes. (f) Histogram of mobility measured from 27 devices. The average and maximum values of mobility are $5.1 \pm 2.7 \mathrm{~cm}^{2} \mathrm{~V}^{-1} \mathrm{~s}^{-1}$ and $11.5 \mathrm{~cm}^{2} \mathrm{~V}^{-1} \mathrm{~s}^{-1}$, respectively. Reproduced from Ref. [32]. Copyright 2014, John Wiley \& Sons, Inc. 
crystal counterpart.

The aforementioned examples suggest that graphene and other van der Waals 2D materials, such as $h$-BN and metal oxides, offer a new type of substrate for high-quality epitaxial growth of various organic semiconductor films for flexible electronic and optoelectronic devices. One of the key challenges in this promising field is the difficulty in the preparation of large-area single-crystal 2D substrate. Although CVD-based method can produce large area of $2 \mathrm{D}$ crystals, the defects and grain boundaries are inevitable, which can cause the disorder/defects in the deposited molecular crystalline films and lead to the deterioration of device performance. Besides, the characterization of thin layer molecular crystals requires special and expensive instruments, such as X-ray diffraction with a synchrotron light source and scanning tunneling microscopy (STM), which are not easily accessible in common laboratories. Nevertheless, van der Waals heterostructures that combine molecular crystals and 2D materials are believed to bring about more exciting research outcomes in the near future.

\section{Received 24 December 2014; accepted 6 January 2015;} published online 16 January 2015

1 Coleman JN, Lotya M, O’Neill A, et al. Two-dimensional nanosheets produced by liquid exfoliation of layered materials. Science, 2011, 331: 568-571

2 Zeng ZY, Yin ZY, Huang X, et al. Single-layer semiconducting nanosheets: high-yield preparation and device fabrication. Angew Chem Int Ed, 2011, 50: 11093-11097

3 Splendiani A, Sun L, Zhang YB, et al. Emerging photoluminescence in monolayer $\mathrm{MoS}_{2}$. Nano Lett, 2010, 10: 1271-1275

4 Novoselov KS, Geim AK, Morozov SV, et al. Electric field effect in atomically thin carbon films. Science, 2004, 306: 666-669

5 Huang X, Yin Z, Wu S, et al. Graphene-based materials: synthesis, characterization, properties, and applications. Small, 2011, 7: 18761902

6 Huang X, Zeng Z, Zhang H. Metal dichalcogenide nanosheets: preparation, properties and applications. Chem Soc Rev, 2013, 42: 1934-1946

7 Novoselov KS, Jiang D, Schedin F, et al. Two-dimensional atomic crystals. Proc Natl Acad Sci USA, 2005, 102: 10451-10453

8 Matte H, Gomathi A, Manna AK, et al. $\mathrm{MoS}_{2}$ and $\mathrm{WS}_{2}$ analogues of graphene. Angew Chem Int Ed, 2010, 49: 4059-4062

9 Niu L, Li K, Zhen H, et al. Salt-assisted high-throughput synthesis of single- and few-layer transition metal dichalcogenides and their application in organic solar cells. Small, 2014, 10: 4651-4657

10 Niu L, Li M, Tao X, et al. Salt-assisted direct exfoliation of graphite into high-quality, large-size, few-layer graphene sheets. Nanoscale, 2013, 5: 7202-7208

11 Zeng Z, Sun T, Zhu J, et al. An effective method for the fabrication of few-layer-thick inorganic nanosheets. Angew Chem Int Ed, 2012, 51: 9052-9056

12 Yin ZY, Li H, Jiang L, et al. Single-layer $\mathrm{MoS}_{2}$ phototransistors. ACS Nano, 2012, 6: 74-80

13 Zhang YJ, Ye JT, Matsuhashi Y, Iwasa Y. Ambipolar $\mathrm{MoS}_{2}$ thin flake transistors. Nano Lett, 2012, 12: 1136-1140

14 He Q, Zeng Z, Yin Z, et al. Fabrication of flexible $\mathrm{MoS}_{2}$ thin-film transistor arrays for practical gas-sensing applications. Small, 2012,
8: 2994-2999

15 Li H, Lu G, Yin ZY, et al. Optical identification of single- and few-layer $\mathrm{MoS}_{2}$ sheets. Small, 2012, 8: 682-686

16 Li H, Yin ZY, He QY, et al. Fabrication of single- and multilayer $\mathrm{MoS}_{2}$ film-based field-effect transistors for sensing no at room temperature. Small, 2012, 8: 63-67

17 Wu S, Zeng Z, He Q, et al. Electrochemically reduced single-layer $\mathrm{MoS}_{2}$ nanosheets: characterization, properties, and sensing applications. Small, 2012, 8: 2264-2270

18 Liu J, Zeng Z, Cao X, et al. Preparation of $\mathrm{MoS}_{2}$-polyvinylpyrrolidone nanocomposites for flexible nonvolatile rewritable memory devices with reduced graphene oxide electrodes. Small, 2012, 8: 3517-3522

19 Huang X, Tan C, Yin Z, Zhang H. 25th anniversary article: hybrid nanostructures based on two-dimensional nanomaterials. Adv Mater, 2014, 26: 2185-2204

20 Shahjamali MM, Bosman M, Cao SW, et al. Gold coating of silver nanoprisms. Adv Funct Mater, 2012, 22: 849-854

21 Huang X, Zeng Z, Bao S, et al. Solution-phase epitaxial growth of noble metal nanostructures on dispersible single-layer molybdenum disulfide nanosheets. Nat Commun, 2013, 4: 1444

22 Schornbaum J, Winter B, Schießl SP, et al. Epitaxial growth of PbSe quantum dots on $\mathrm{MoS}_{2}$ nanosheets and their near-infrared photoresponse. Adv Funct Mater, doi: 10.1002/adfm.201400330

23 Chung $\mathrm{K}$, Lee $\mathrm{CH}$, Yi GC. Transferable gan layers grown on $\mathrm{ZnO}$-coated graphene layers for optoelectronic devices. Science, 2010, 330: 655-657

24 Tan CL, Zeng ZY, Huang X, et al. Liquid-phase epitaxial growth of two-dimensional semiconductor hetero-nanostructures. Angew Chem Int Ed, doi: 10.1002/anie. 201410890

25 Forrest SR. Ultrathin organic films grown by organic molecular beam deposition and related techniques. Chem Rev, 1997, 97: 1793-1896

26 Harada Y, Ozaki H, Ohno K. Selective observation of outermost surface layer during epitaxial growth by penning-ionization electron spectroscopy: pentacene on graphite. Phys Rev Lett, 1984, 52: 2269-2272

27 Lee WH, Park J, Sim SH, et al. Surface-directed molecular assembly of pentacene on monolayer graphene for high-performance organic transistors. J Am Chem Soc, 2011, 133: 4447-4454

28 Colson JW, Woll AR, Mukherjee A, et al. Oriented 2D covalent organic framework thin films on single-layer graphene. Science, 2011, 332: $228-231$

29 Koma A. Van der Waals epitaxy-a new epitaxial growth method for a highly lattice-mismatched system. Thin Solid Films, 1992, 216: $72-76$

30 He D, Zhang Y, Wu Q, et al. Two-dimensional quasi-freestanding molecular crystals for high-performance organic field-effect transistors. Nat Commun, 2014, 5: 5162

31 Lee CH, Schiros T, Santos EJG, et al. Epitaxial growth of molecular crystals on van der waals substrates for high-performance organic electronics. Adv Mater, 2014, 26: 2812-2817

Acknowledgements This work was supported by Ministry of Education of Singapore under AcRF Tier 2 (ARC 26/13, MOE2013-T2-1-034), AcRF Tier 1 (RG 61/12, RGT18/13, and RG5/13), Start-Up Grant (M4080865.070.706022) in Singapore, the National Natural Science Foundation of China (51322202), and Natural Science Foundation of Jiangsu Province (BK20130927). This research is also conducted by NTU-HUJ-BGU Nanomaterials for Energy and Water Management Programme under the Campus for Research Excellence and Technological Enterprise, that is supported by the National Research Foundation, Prime Minister's Office, Singapore. 
Author contributions Huang $\mathrm{X}$ and Zhang $\mathrm{H}$ contributed to the general discussion and wrote the manuscript together.
Conflict of interest The authors declare that they have no conflict of interest.

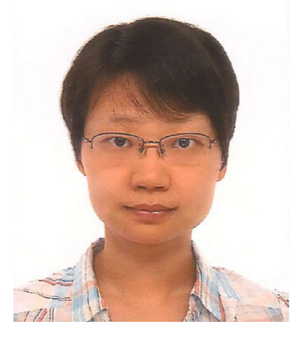

Xiao Huang received her bachelor degree from the School of Materials Science and Engineering of Nanyang Technological University in Singapore in 2006 and completed her PhD under the supervision of Professor Hua Zhang and Professor Freddy Boey in 2011. After that, she worked as a postdoctoral fellow in Professor Hua Zhang's group. She is now a professor at the Institute of Advanced Materials of Nanjing Tech University in China. Her research interest includes the synthesis and applications of two-dimensional nanomaterial-based hybrid structures.

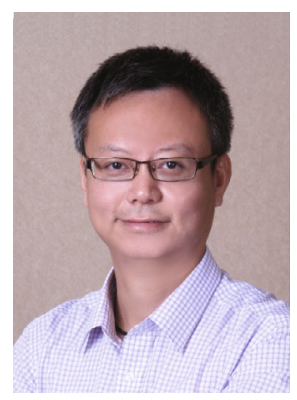

Hua Zhang obtained his BSc and MSc degrees at Nanjing University in 1992 and 1995, respectively, and completed his PhD supervised by Professor Zhongfan Liu at Peking University in 1998. As a Postdoctoral Fellow, he joined Prof. Frans C. De Schryver's group at Katholieke Universiteit Leuven (Belgium) in 1999, and then moved to Prof. Chad A. Mirkin's group at Northwestern University in 2001. After he worked at NanoInk Inc. (USA) and Institute of Bioengineering and Nanotechnology (Singapore), he joined Nanyang Technological University in July 2006. He was promoted to a tenured Associate Professor in March 2011, and Full Professor in September 2013. His current research interests focus on synthesis of two-dimensional nanomaterials and carbon materials (graphene and carbon nanotubes), and their applications in nano- and bio-sensors, nanoelectronics, clean energy, water remediation, etc.

中文摘要 通过剥离以石墨烯、金属硫族化合物等为代表的基于范德华作用力形成的层状晶体而得到的二维无机纳米片材料, 目前 已经被用作外延生长的模板, 广泛地应用于具有特定晶格取向和晶体表面的无机纳米晶体的制备. 此外, 基于石墨烯和六方氮化硼二 维材料的半导体分子晶体的制备正被不断报道, 成为一个新的研究热点. 这种基于二维材料的分子晶体复合物在有机电子器件方面具 有巨大的应用前景. 本文对基于二维材料的分子晶体复合材料的制备及应用进行了总结, 同时也讨论了这个领域目前面临的问题和挑 战, 以及未来的研究方向. 